\title{
La inteligencia interpersonal y la convivencia armoniosa en estudiantes de secundaria de la I.E. 109, Lima 2020
}

\section{Interpersonal intelligence and harmonious coexistence in high school students from the I.E.109, Lima 2020}

\author{
Rocío Nauca ${ }^{1 \mathbb{D} *}$, Sebastián Sánchez ${ }^{2}$ (i) , Carlos Sevilla ${ }^{3(\mathbb{D})}$ y Eufemia Martinez ${ }^{(\mathbb{D}}$
}

\begin{abstract}
RESUMEN
El objetivo de la presente investigación fue, determinar la relación que existe entre la inteligencia interpersonal y la convivencia armoniosa en estudiantes de secundaria de la I.E. 109, Lima. Esta investigación fue de tipo aplicada, su diseño no experimental, transversal, correlacional y enfoque cuantitativo. La muestra fue constituida por 80 estudiantes de secundaria de la I.E 109; se empleó la técnica de muestreo estratificado. Los instrumentos fueron previamente validados por expertos; de igual forma para determinar la confiabilidad se aplicó pruebas pilotos. Posteriormente a la recolección y procesamiento de datos, se realizó el análisis estadístico, en donde se estableció una relación de r=-0.108 entre las variables: inteligencia interpersonal y convivencia armoniosa. Este grado de correlación identificó que la relación entre las dos variables es inversa y escasa y no hay evidencia suficiente que relacione las dos variables. En cuanto a la significancia bilateral fue $\mathrm{P}=0.342$ mayor a 0.05 lo que indicó que la relación no es significativa.
\end{abstract}

Palabras clave: Inteligencia interpersonal, convivencia armoniosa, conflicto.

\begin{abstract}
The objective of the present investigation was to determine the relationship between interpersonal intelligence and harmonious coexistence in secondary school students from I.E. 109, Lima. This research was of applied type, its non-experimental, transversal, correlational design and quantitative approach. The sample was constituted by 80 high school students of the I.E. 109; the technique of stratified sampling was used. The instruments were previously validated by experts; in the same way, pilot tests were applied to determine their reliability. After the collection and processing of data, the statistical analysis was carried out, where a relation of $\mathrm{r}=-0.108$ was established among the variables: interpersonal intelligence and harmonious coexistence. This degree of correlation indicated that the relationship between the two variables is inverse and scarce, and there is not enough evidence to relate the two variables. As for bilateral significance, $\mathrm{P}=0.342$ greater than 0.05 indicated that the relationship is not significant.
\end{abstract}

Keywords: interpersonal intelligence, harmonious coexistence, conflict.

DOI: https://doi.org/10.37787/pakamuros-unj.v8i3.137

Recibido: 30/09/2020. Aceptado: 17/10/2020

* Autor para correspondencia

1. Universidad César Vallejo, Perú. Email: rocioandrea8800@gmail.com

2. Universidad César Vallejo, Perú. Email: ssanchez@ucv.edu.pe

3. Universidad César Vallejo, Perú. Email: carlossevillam67@ gmail.com

4. Universidad César Vallejo, Perú. Email: eufemiamr2@gmail.com 


\section{INTRODUCCIÓN}

En nuestros tiempos podemos percibir que los países a nivel mundial enfrentan una gran problemática causada de alguna manera por la modernidad de la tecnología en sus variadas formas de comunicación y contacto personal. Toda esta situación repercute en la sociedad originando diversas consecuencias que se manifiestan en la conducta de los seres humanos en sus diferentes ámbitos en los que se desenvuelve diariamente; estos conflictos que surgen por malos entendidos y también por no saber utilizar adecuadamente la inteligencia interpersonal en nuestro diario vivir.

Las familias se han visto afectadas de alguna manera, lo que origina diversos cambios tanto a nivel familiar y social. La escuela también se representa como un hogar donde se puede apreciar el modo de convivencia entre alumnos, profesores y padres; observándose situaciones que afectan la buena convivencia en armonía presentándose casos de acoso, violencia y maltrato que sufren algunos estudiantes por sus compañeros. Este problema radica principalmente por las relaciones interpersonales debilitadas evidenciándose en los estudiantes con dificultades de relación armoniosa y comunicación asertiva. En su estudio, Díaz (2015) sostuvo basado en otras teorías la importancia de esta inteligencia en lo emocional, la cual influye en lo académico.

Toda esa situación también la sostiene Masabanda (2017), donde destacó que los estudiantes tienen dificultades en manifestar diversas situaciones, que implica el dominio de la inteligencia interpersonal y la poca importancia que se le ha dado por enfocarse en otros aspectos.

Espilco (2019), manifiesta que no existen barreras en el tiempo para que un individuo pueda triunfar y resolver sus propios conflictos que le presenta la vida; para eso debe desarrollar su parte cognitiva y desarrollar la inteligencia personal. Es por eso que en las instituciones educativas se debe promover actividades que permitan vivir experiencias agradables y significativas para que los estudiantes al poder interactuar con sus pares fortalezcan una relación de convivencia sana que les ayude a tener una vida en armonía con los demás.

El escenario de la I.E. № 109 ha mostrado un ambiente de conflictos escolares que pasaba por la falta de respeto a docentes y entre pares, con una conducta disruptiva que lindaba con el desorden psicológico. Razón por la que se pretendió aportar con esta investigación sobre la inteligencia interpersonal y la convivencia armoniosa alternativas para enmendar esta problemática.

De lo expuesto para tomar en cuenta en la presente investigación se formuló la siguiente pregunta que será el problema general ¿De qué forma se relaciona la inteligencia interpersonal y la convivencia armoniosa en los estudiantes de secundaria de la Institución Educativa 109, Lima ?, de este problema general tenemos los problemas específicos ¿De qué manera se relaciona la dimensión de empatía y la convivencia armoniosa en los estudiantes de la I.E 109; ¿Cuál es la relación entre la dimensión de 
asertividad y la convivencia armoniosa en los estudiantes de la I.E 109 ?; ¿Cuál es la relación entre la dimensión de resolución de conflictos y la convivencia armoniosa en los estudiantes de la I.E 109 ?

Para la presente investigación se formulan los siguientes objetivos: objetivo general: OG. Establecer la relación entre la inteligencia interpersonal y la convivencia armoniosa en los estudiantes de la IE 109, Lima. Y como objetivos específicos: OE1. Determinar la relación entre la dimensión de empatía y la convivencia armoniosa en los estudiantes de la IE 109 Inca Manco Cápac, 2020. OE2. Determinar la relación entre la dimensión de asertividad y la convivencia armoniosa en los estudiantes de la IE 109 Inca Manco Cápac, 2020. OE3. Determinar la relación entre la dimensión de resolución de conflictos y la convivencia armoniosa en los estudiantes de la IE 109 Inca Manco Cápac, 2020.

En este sentido se plantea la hipótesis general: $\mathrm{Hg}$ : Existe relación significativa entre la inteligencia interpersonal y la convivencia armoniosa en los estudiantes de la I.E 109, Lima. Y como hipótesis específica: H1: Existe relación significativa entre la dimensión de empatía y la convivencia armoniosa en los estudiantes de la I.E 109, Lima. H2: Existe relación significativa entre la dimensión de asertividad y la convivencia armoniosa en los estudiantes de la I.E 109, Lima. H3: Existe relación significativa entre la dimensión resolución de conflictos y la convivencia armoniosa en los estudiantes de la I.E 109, Lima.

\section{MATERIALES Y MÉTODOS}

\section{Tipo y diseño de investigación}

Tipo de investigación: La tipología del estudio empleada se basa en Valderrama (2015), sostiene que este tipo de investigación se refiere a la forma de indagar con el fin de resolver una problemática. Las variables estudiadas, fue la inteligencia interpersonal, y la convivencia armoniosa. El cuestionario estuvo compuesto por 25 ítems y una escala tipo Likert.

\section{Población, muestra y muestreo}

\section{Población}

Estuvo compuesta por 100 estudiantes de secundaria de la institución educativa número 109 Inca Manco Cápac, los Olivos, Lima 2020.

\section{Muestra}

Estuvo conformada por 80 estudiantes de secundaria de a la institución educativa número 109 Inca Manco Cápac, los Olivos, Lima 2020.

\section{Muestreo}

El tipo de muestreo empleado fue probabilístico y aleatorio.

\section{Instrumentos de recolección de datos, validez y confiabilidad}




\section{Instrumento}

El instrumento usado para administrar la información de las variables fue el cuestionario definido por Carrasco (2018), como aquello de mayor utilización en los estudios de investigación, y cuando existe poblaciones numerosas, la cual nos permite el recojo de datos de manera directa mediante interrogantes que son respondidas de manera individual. Se empleó como instrumento el cuestionario.

Ficha técnica 1:

Variable: Inteligencia interpersonal.

Autora: Br. Olivia Espilco Vilcapuma

Adaptado: Br. Rocío Nauca Guzmán

Procedencia: Perú

Administración: Colectiva

Duración: 20 minutos

Dimensiones: Empatía, Asertividad y resolución de conflictos

$\mathrm{N}^{\circ}$ de ítems: 25 ítems

Aplicación: Adolescentes escolares hombres y mujeres

Ficha técnica 2:

Variable: Convivencia armoniosa

Autora: Br. Olivia Espilco Vilcapuma

Adaptado: Br. Rocío Nauca Guzmán

Procedencia: Perú

Administración: Colectiva

Duración: 20 minutos

Dimensiones: Clima positivo, Práctica de la disciplina y Participación activa.

$\mathrm{N}^{\circ}$ de ítems: 25 ítems

Aplicación: Adolescentes escolares hombres y mujeres

\section{Validez}

Los cuestionarios fueron validados a traves del juicio de expertos (crítica de jueces), con la apreciación de 3 profesionales expertos en el área de educación y docencia universitaria con el grado académico de maestro y doctor para establecer si los items se encuentran en correspondencia con las dimensiones propuestas y los indicadores de las mismas, asimismo con la variable de estudio (Tabla 1). 
Tabla 1. Validez de contenido de los instrumentos de inteligencia interpersonal y convivencia armoniosa

\begin{tabular}{cll}
\hline Experto & Nombre y Apellidos & Opinión \\
\hline 1 & Dr. Johnny Félix Farfán Pimentel & Aplicable \\
2 & Dra. Yolanda Josefina Huayta Franco & Aplicable \\
3 & Mg. Rommel Lizandro Crispín & Aplicable \\
\hline
\end{tabular}

\section{Confiabilidad}

Para Arribas (2008), la confiabilidad es "cuando un instrumento logra medir con precisión, no cayendo en errores. Es decir que al ser aplicados de manera repetida sus resultados se mostrarán en condiciones similares e idénticas" (p. 27). Por otra parte Frias (2019), manifestó que la fiabilidad son las puntuaciones en los intrumentos de medida.

Para establecer la confiabilidad del instrumento se aplicó una prueba piloto a 20 estudiantes con similares caracteristicas a la muestra seleccionada, se tabularon los datos y se analizaron por medio del método de consitencia interna (coeficiente de alfa de Cronbach) utilizando el programa estadistico SPSS V.25. Para fijar la regla de valoración se consideró lo señalado por, Hernández,Fernández y Baptista(2018): Un coeficiente por arriba de 0.75 revela que el instrumento es confiable (Tabla 2).

Tabla 2. Estadístico de fiabilidad del instrumento Inteligencia interpersonal.

\begin{tabular}{cc}
\multicolumn{2}{c}{ Estadísticas de fiabilidad } \\
\hline Alfa de Cronbach & N de elementos \\
.899 & 25 \\
\hline
\end{tabular}

Interpretación: En la Tabla 2, se aprecia que el coeficiente del alfa de Cronbach es de 0,899 lo que significa que el instrumento tiene una confiabilidad de nivel bueno.

Tabla 3. Estadístico de fiabilidad del instrumento convivencia armoniosa

\begin{tabular}{cc}
\hline \multicolumn{2}{c}{ Estadísticas de fiabilidad } \\
\hline Alfa de Cronbach & N de elementos \\
.853 & 25 \\
\hline
\end{tabular}

Interpretación: Según la Tabla 3, se percibe que el coeficiente del alfa de Cronbach es de 0,853 lo que significa que el instrumento tiene una confiabilidad de nivel bueno. 


\section{Procedimiento}

Como primera actividad del procedimiento de recolección de datos se efectuó la aplicación de una prueba piloto cuyo propósito fue determinar qué tan confiable son los instrumentos elaborados para medir las variables de estudio. Esta prueba se efectuó en una población similar a la de la población de estudio.

\section{Métodos de análisis de datos}

En el análisis y procesamiento de los hallazgos se usó el SPSS cuya versión fue veinticinco y a través de ello se procedió con la prueba no paramétrica coeficiente de correlación Rho de Spearman con el objetivo de encontrar la correspondencia de las variables de estudio.

\section{Aspectos éticos}

Los principios éticos se basaron en la relación ética con el problema investigado con las personas que participaron, reconociendo los principios universales para todos los sujetos partiendo desde la participación voluntaria y guardando la confidencialidad y respeto en el contexto en el cual se produjo la investigación.

\section{RESULTADOS}

Se ha realizado el análisis descriptivo de las variables y dimensiones.

Tabla 4. Distribución de la frecuencia y porcentajes de la variable inteligencia interpersonal

\begin{tabular}{cccccc}
\hline \multicolumn{6}{c}{ Inteligencia interpersonal } \\
\hline & Frecuencia & Porcentaje & Porcentaje válido & $\begin{array}{c}\text { Porcentaje } \\
\text { acumulado }\end{array}$ \\
\hline \multirow{2}{*}{ Válido } & Proceso & 10 & 12,5 & 12,5 & 12,5 \\
& Logro & 70 & 87,5 & 87,5 & 100,0 \\
& Total & 80 & 100,0 & 100,0 & \\
\hline
\end{tabular}

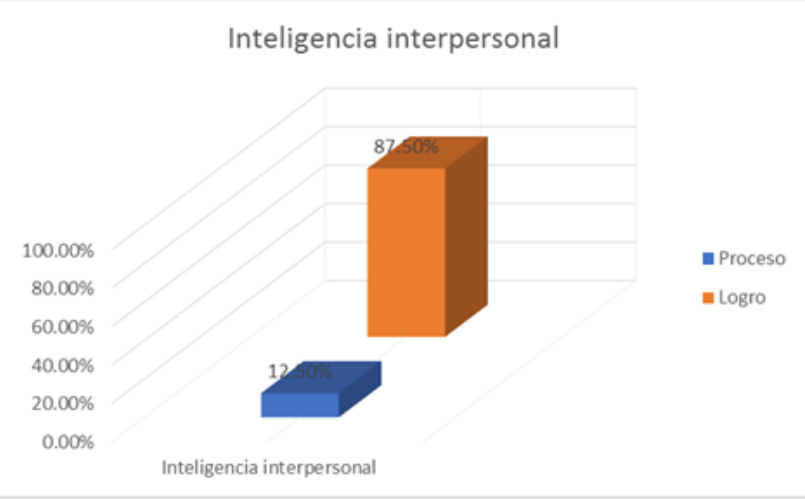

Figura 1. Distribución de la frecuencia y porcentajes de la variable inteligencia interpersonal. 
Interpretación: De la Tabla 4 y Figura 1, se observa que los estudiantes encuestados expresaron que la inteligencia interpersonal está en un nivel de proceso alcanzando el $12.5 \%$, mientras que un $87.5 \%$ alcanzó el nivel de logro. Apreciándose de los resultados que la predominancia de la variable inteligencia personal es de nivel de logro.

Tabla 5. Distribución de la frecuencia y porcentajes de la variable convivencia armoniosa

\begin{tabular}{crcccc}
\hline \multicolumn{5}{c}{ Convivencia armoniosa } \\
\hline Válido & Regular & 6 & 7,5 & 7,5 & $\begin{array}{c}\text { Porcentaje } \\
\text { acumulado }\end{array}$ \\
& Bueno & 74 & 92,5 & 92,5 & 100,0 \\
& Total & 80 & 100,0 & 100,0 & \\
\hline
\end{tabular}

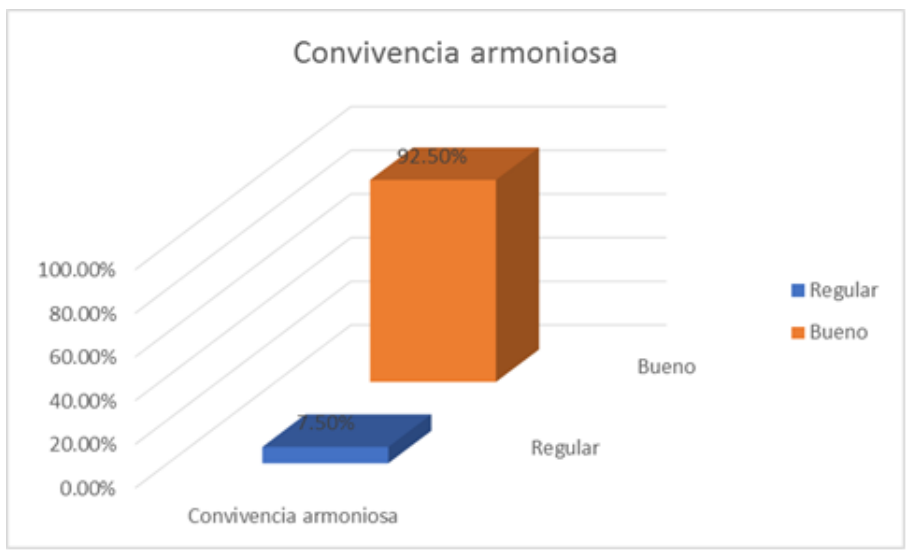

Figura 2. Distribución de la frecuencia y porcentajes de la variable convivencia armoniosa.

Interpretación: De la Tabla 5 y Figura 2, se observa que los estudiantes encuestados manifestaron que el $7.5 \%$ alcanzaron una convivencia armoniosa de regular, mientras que un $92.5 \%$ expresó que tiene una convivencia armoniosa de nivel bueno. Apreciándose de los resultados que la predominancia de la convivencia armoniosa es de nivel bueno.

Tabla 6. Niveles de comparación entre las variables inteligencia interpersonal y convivencia armoniosa

Tabla cruzada Inteligencia interpersonal*Convivencia armoniosa

\begin{tabular}{rrrrrr}
\hline & & \multicolumn{2}{c}{$\begin{array}{c}\text { Convivencia armoniosa } \\
\text { regular }\end{array}$} & bueno & Total \\
\hline \multirow{2}{*}{ Inteligencia interpersonal } & \multirow{2}{*}{ Proceso } & Recuento & 0 & 10 & 10 \\
\cline { 3 - 6 } & & $\%$ del total & $0,0 \%$ & $12,5 \%$ & $12,5 \%$ \\
& \multirow{2}{*}{ Logro } & Recuento & 6 & 64 & 70 \\
& & $\%$ del total & $7,5 \%$ & $80,0 \%$ & $87,5 \%$ \\
& \multirow{2}{*}{ Total } & Recuento & 6 & 74 & 80 \\
& & $\%$ del total & $7,5 \%$ & $92,5 \%$ & $100,0 \%$ \\
\hline
\end{tabular}




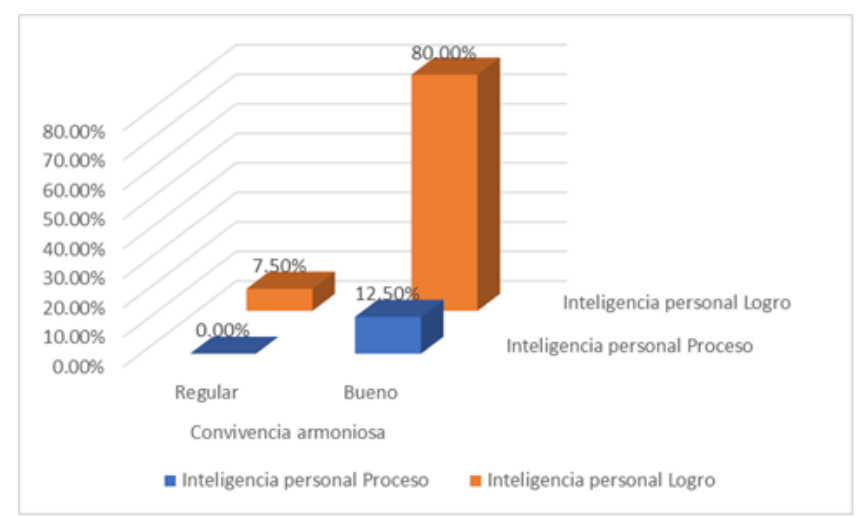

Figura 3. Nivel de comparación entre las variables inteligencia interpersonal y convivencia armoniosa.

Interpretación: Sobre la variable inteligencia interpersonal cruzada con la convivencia armoniosa, en la Tabla 6 y Figura 3, se observa que cuando la inteligencia interpersonal está en nivel proceso de $12.5 \%$; la convivencia armoniosa alcanzó el nivel de bueno; y cuando la inteligencia interpersonal está en nivel de logro el 7.5\%, la convivencia armoniosa alcanzó el nivel de regular; mientras que el $80.0 \%$ de los encuestados considera que la inteligencia interpersonal está en un nivel de logro, la convivencia armoniosa está en un nivel bueno.

\section{Análisis inferencial}

\section{Prueba de hipótesis general}

Ho: No existe relación significativa entre la inteligencia personal y la convivencia armoniosa

Ha: Existe relación significativa entre la inteligencia personal y la convivencia armoniosa

Decisión estadística

$\mathrm{P}>0,05=$ acepta hipótesis nula $(\mathrm{Ho})$

$\mathrm{P}<0,05=$ rechaza hipótesis nula $(\mathrm{Ha})$

Nivel de significancia: $95 \%$ de confianza.

Prueba estadística: no paramétrica, coeficiente Rho de Spearman (Tabla 7).

Tabla 7. Coeficiente de correlación de Spearman de las variables: Inteligencia interpersonal y la convivencia armoniosa

\begin{tabular}{ccrr}
\hline & & $\begin{array}{r}\text { Inteligencia } \\
\text { interpersonal }\end{array}$ & $\begin{array}{r}\text { Convivencia } \\
\text { armoniosa }\end{array}$ \\
\hline Rho de Spearman & Inteligencia interpersonal & Coeficiente de & 1,000 \\
& & correlación &,- 108 \\
& Sig. (bilateral) & $\mathrm{N}$ & 80 \\
\hline
\end{tabular}




\begin{tabular}{rrrr}
\hline Convivencia armoniosa & $\begin{array}{r}\text { Coeficiente de } \\
\text { correlación }\end{array}$ &,- 108 & 1,000 \\
& Sig. (bilateral) &, 342 & . \\
& $\mathrm{N}$ & 80 & 80 \\
\hline
\end{tabular}

\section{Descripción del grado de relación entre las variables}

Los resultados del análisis estadístico señalaron que existe una relación de $\mathrm{r}=-0.108$ entre las variables: inteligencia interpersonal y convivencia armoniosa. Este resultado demostró que la relación es inversa con un nivel de correlación escasa entre las variables de esta investigación.

\section{Decisión estadística}

La significancia de $\mathrm{p}=0,342$ determinó que $\mathrm{p}>0,05$ lo cual indicó que no hay relación significativa entre las variables, en consecuencia, se acepta la hipótesis nula y se rechaza la hipótesis alterna

\section{DISCUSIÓN}

Los resultados obtenidos en la descripción estadística mostraron una correlación con $\mathrm{r}=-0.108$, que indicó una relación inversa escasa entre las variables inteligencia interpersonal y convivencia armoniosa; con una significancia de $\mathrm{p}=0.342$ mayor a 0.05 lo que determinó que no hay una relación significante entre estas dos variables. A partir de estos resultados, se rechazó la hipótesis alterna y se aceptó la hipótesis nula. Sin embargo, estos resultados no guardaron mucha relación con Espilco, (2019) que en su investigación concluyó que a mayor nivel de convivencia escolar mejor desarrollo de la inteligencia interpersonal. Y de la misma manera se diferenció con Medina, (2016) que según sus estudios concluyó que hay una relación importante entre las variables: Inteligencia interpersonal y clima escolar, siendo de valor moderado, cuyo valor es $\mathrm{p}=.000(\mathrm{p}<.01)$.

Los resultados obtenidos mostraron una correlación con $\mathrm{r}=-0.069$; que indicó que la relación es inversa y escasa entre la dimensión empatía y la variable convivencia armoniosa; con una significancia de 0,543 mayor a 0,05 determinando que no hay relación significativa entre las variables empatía y la convivencia armoniosa; aceptándose la hipótesis nula y rechazando la alterna. Resultados que se contradijeron con Rojas, (2018) el cual concluyó que para que exista una convivencia armoniosa en el aula de clase deben originarse adecuadas relaciones personales. Y con López et al., (2018) en su estudio expresaron que la empatía es considerada un talento que poseen las personas y se evidencian cuando se ponen en lugar de otro, cuando se preocupas por los demás, se solidarizan con sus semejantes y se manifiesta con la demostración de las acciones. 
De los resultados obtenidos en el estudio se mostró una correlación de r= - 0,114, que indicó una relación inversa escasa entre la dimensión de asertividad y la convivencia armoniosa; asimismo presentó una significancia de $\mathrm{p}=0,316$ mayor a 0,05 y que no existió relación significativa entre estas variables, en consecuencia, se aceptó la hipótesis nula y se rechazó la hipótesis alterna. Estos hallazgos se comparan con lo que afirmó Ángeles, (2017) el asertividad es una conducta o comportamiento y este se da en medio de la comunicación humana, la cual permite expresar lo que se piensa, siente y desea de una mejora manera, clara y oportuna. Y según Tafur, (2016) expresó que una acción ejecutada con asertividad permite al sujeto desenvolverse de forma correcta, teniendo en cuenta sus metas para alcanzarlas y siendo respetuoso del derecho ajeno, pero si no se considera esto y actuamos sin reflexionar se torna esta acción un poco violenta.

De los resultados del análisis estadístico se mostró una correlación de r= 0,149, que indicó una relación positiva escasa entre la resolución de conflictos y la convivencia armoniosa, con un nivel de significancia de $\mathrm{p}=0,186$ la cual es mayor a 0,05, lo que determinó que no hay relación significativa entre estas variables. En consecuencia, se aceptó la hipótesis nula y se rechazó la hipótesis alterna. Sin embargo, estos resultados se contraponen con Minedu, (2013) que manifestó que el conflicto al resolverse dentro de un marco del diálogo, nos permite entender y reforzar diversas acciones como: el compromiso, la empatía, la consideración, el ser asertivo y comunicación horizontal, siendo aspectos elementales para el accionar de los alumnos y la edificación de una coexistencia equilibrada. Por otra parte, Laureano, (2018) en su trabajo concluyó que según los hallazgos obtenidos producto de esta investigación tuvo un Rho de Spearman de 0,432, existiendo una correspondencia media y positiva entre sus variables convivencia escolar y habilidades sociales.

\section{CONCLUSIONES}

Según los resultados descriptivos en la tabla cruzada se observó que al comparar las dos variables de estudio no hay una proporcionalidad directa entre ambas variables, la relación entre ellas solo se puede evidenciar en el nivel de logro para la inteligencia interpersonal y de bueno la convivencia armoniosa. Por otro lado, los resultados obtenidos en la parte inferencial, se mostró una relación r=-0.108 determinándose una correlación escasa e inversa entre las variables la inteligencia interpersonal y la convivencia armoniosa, ello sugiere que la variable convivencia armoniosa depende de otras variables y no necesariamente con la inteligencia interpersonal.

En cuanto a la relación con las dimensiones de la variable inteligencia interpersonal se evidenció un r= -0,069 entre la dimensión empatía y la variable convivencia armoniosa señalándose que la relación es inversa con un nivel de correlación escasa. Presentó un p valor de 0,543 el cual es mayor a 0,05; lo que 
indicó que no hay relación significativa entre estas variables, por lo tanto, se aceptó la hipótesis nula y se rechazó la alterna.

Se detalla también en los resultados de este estudio que existe una relación $\mathrm{r}=-$ 0, 114 entre la dimensión asertividad y la convivencia armoniosa, lo que señaló que existe una relación inversa con un nivel de correlación escasa entre estas variables. Y en cuanto al p valor presentó un valor de 0,316, mayor a 0,05 por lo que se concluye, que no hay relación significativa entre estas variables, aceptándose la hipótesis nula y se rechaza la hipótesis alterna.

Por otro lado, se obtuvo una relación de $\mathrm{r}=0,149$ entre resolución de conflictos y convivencia armoniosa. Este resultado señaló que la relación es positiva con un nivel de correlación escasa entre las variables mencionadas. En cuanto con un p valor de 0,186, mayor a 0,05 lo cual indica, que no hay relación significativa entre las variables, por lo que, se acepta la hipótesis nula y se rechaza la alterna.

\section{REFERENCIAS BIBLIOGRÁFICAS}

Ángeles, R. (2017). El asertividad y el desarrollo de la convivencia en estudiantes universitarios. (Tesis de maestría). Universidad de San Martín de Porres. Lima, Perú. Recuperado de:

http://www.repositorioacademico.usmp.edu.pe/bitstream/handle/usmp/2792/angeles_hrb.pdf;jsessionid =1A77894D44EBCA180949044B7F88B46E? sequence $=3$

Carrasco, D. S. (2018). Metodología de la investigación científica. Pautas metodológicas para diseñar y elaborar el proyecto de investigación. Editorial San Marcos. Lima.

Díaz, N. (2015). Relación entre las inteligencias intrapersonal e interpersonal y el rendimiento escolar: Una propuesta. (Tesis de maestría). Universidad La Rioja. España. Recuperado de: https://reunir.unir.net/bitstream/handle/123456789/3486/DIAZ\%20GIRALDO\%2C\%20NAZA RETpdf? sequence $=1 \&$ is Allowed $=\mathrm{y}$

Díaz, S. (2018) Capacidades docentes para promover una convivencia armoniosa en la Institución educativa N ${ }^{\circ}$ 10796" Carlos Augusto Salaverry"- Distrito La Victoria- Chiclayo- 20172018.Pontificia Universidad Católica del Perú. Recuperado de: http://hdl.handle.net/20.500.12404/10783

Espilco, O. (2019). Convivencia escolar y la inteligencia interpersonal en estudiantes del V ciclo en la IE. Casa Blanca de Jesús, SJL - 2019. (Tesis de maestría). Universidad César Vallejo. Lima. Perú. Recuperado de: http://repositorio.ucv.edu.pe/bitstream/handle/UCV/40657/ESPILCO_VO.pdf?sequence=1\&is Allowed $=\mathrm{y}$ 
Frías, D. (2019). Apuntes de consistencia interna de las puntuaciones de un instrumento de medida. Universidad de Valencia. España. Recuperado de: https://www.uv.es/friasnav/AlfaCronbach.pdf

Hernández, R. Fernández, C. y Baptista, P. (2018). Metodología de la Investigación. Sexta Edición. Editorial McGraw Hill. Recuperado de: http://observatorio.epacartagena.gov.co/.

Laureano, C. (2018). Convivencia escolar y habilidades sociales en estudiantes de $2^{\circ}$ grado de secundaria en Instituciones educativas del distrito de San Martin de Porres - 2018. (Tesis de maestría). Universidad César Vallejo, Lima, Perú.

López Pérez, B., Fernández Pinto I. y Abad F. (2008), Test de empatía cognitiva y afectiva. Madrid, España: Ed. TEA Ediciones, SA.

Masabanda, E. (2017). Inteligencia Interpersonal en los estudiantes de la Unidad Educativa Cap. Edmundo Chiriboga de la ciudad de Riobamba durante el período académico 2016-2017. (Tesis de grado). Universidad Nacional de Chimborazo. Ecuador. Recuperado de http://dspace.unach.edu.ec/handle/51000/3939

Medina, C. (2016). Inteligencia interpersonal y clima escolar en estudiantes de primero de secundaria Institución Educativa "Ramiro Prialé”, San Juan de Lurigancho. 2015. Tesis de Maestría. Recuperado de.http://repositorio.ucv.edu.pe/bitstream/handle/20.500.12692/5852/MEDINA_YE.pdf?sequen ce $=4 \&$ is Allowed $=\mathrm{y}$

Ministerio de Educación (2013). Aprendiendo a resolver conflictos en las instituciones educativas: orientaciones para directivos $\mathrm{y}$ tutores de primaria y secundaria. Recuperado de http://repositorio.minedu.gob.pe/handle/123456789/4453

Rojas, Y. (2018) Convivencia armoniosa dentro del aula: plan de acción. I.E $\mathrm{N}^{\circ} 307$ del distrito de Chalaco, provincia de Morropón, región Piura. Pontificia Universidad Católica del Perú. Recuperado de: http://hdl.handle.net/20.500.12404/10783

Tafur, J. (2016). Clima social familiar y asertividad en alumnos de cuarto y quinto año de secundaria de un colegio estatal de la esperanza, Trujillo. (Tesis de grado). Universidad Privada Antenor Orrego. Trujillo, Perú. Recuperado de:

http://repositorio.upao.edu.pe/bitstream/upaorep/2461/1/re_psico_juiliana.tafur_clima.social.familiar.y. asertividad.en.alumnos.de.cuarto.y\%20quinto.a\%c3\%91o_datos.pdf

Valderrama S. (2015). Pasos para elaborar proyectos y tesis de investigación. Perú: Editorial San Marcos - Lima 